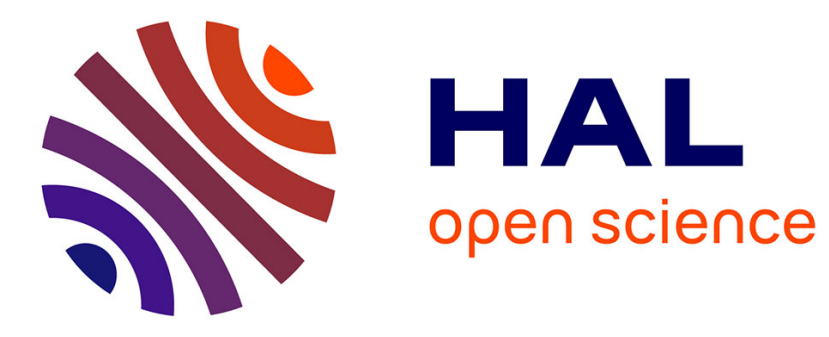

\title{
Color characterization methods for a multispectral camera
}

\author{
Haris Ahmad Khan, Phil Green
}

\section{To cite this version:}

Haris Ahmad Khan, Phil Green. Color characterization methods for a multispectral camera. International Symposium on Electronic Imaging 2018: Color Imaging XXIII: Displaying, Processing, Hardcopy, and Applications, IS\&T Jan 2018, San Francisco, United States. 10.2352/ISSN.24701173.2018.16.COLOR-221 . hal-01709045

\section{HAL Id: hal-01709045 \\ https://hal.science/hal-01709045}

Submitted on 14 Feb 2018

HAL is a multi-disciplinary open access archive for the deposit and dissemination of scientific research documents, whether they are published or not. The documents may come from teaching and research institutions in France or abroad, or from public or private research centers.
L'archive ouverte pluridisciplinaire HAL, est destinée au dépôt et à la diffusion de documents scientifiques de niveau recherche, publiés ou non, émanant des établissements d'enseignement et de recherche français ou étrangers, des laboratoires publics ou privés. 


\title{
Color characterization methods for a multispectral camera
}

\author{
Haris Ahmad Khan ${ }^{1,2}$ and Phil Green ${ }^{1}$ \\ ${ }^{1}$ The Norwegian Colour and Visual Computing Laboratory, \\ NTNU - Norwegian University of Science and Technology, Gjøvik, Norway \\ ${ }^{2}$ Le2i, FRE CNRS 2005, Univ. Bourgogne Franche-Comté, Dijon, France
}

\begin{abstract}
With advances in sensor technology, the availability of multispectral cameras and their use are increasing. Having more information compared to a three-channel camera has its advantages but the data must be handled appropriately. In this work, we are interested in multispectral camera characterization. We measure the camera characterization performance by two methods, by linear mapping and through spectral reconstruction. Linear mapping is used in 3-channel camera characterization and we use the same method for a multispectral camera. We also investigate whether instead of linear mapping, spectral reconstruction from the camera data improves the performance of color reproduction. The recovery of reflectance spectra is an under-determined problem and certain assumptions are required for obtaining a unique solution. Linear methods are generally used for spectral reconstruction from the camera data and are based on training on known spectra. These methods can perform well when the test data consists of a subset of the training spectra, however, their performance is significantly reduced when the test data is different. In this paper, we also investigate the role of training spectra for camera characterization. Five different spectral reflectance datasets are used for training the camera characterization models. Finally we provide a comparison between the linear mapping and spectral reconstruction methods for multispectral camera characterization and also test the camera characterization framework on hyperspectral images of natural scenes.
\end{abstract}

\section{Introduction}

A common procedure for obtaining device independent color information from a digital camera is to characterize the camera in terms of CIE tristimulus values $[1,2,3,4,5,6]$. Ideally, the spectral sensitivities of a digital camera with three color channels would satisfy the Luther condition, in which it is assumed that they are linear transformation of the CIE color matching function [7]. However, real cameras do not follow the Luther condition because of manufacturing constraints and limitations in design. Because of this issue, device metamerism may occur, where two surfaces with different spectral behavior produce identical camera response when imaged under the same illumination and hence provide the same XYZ values. Such metamerism limits the colorimetric application of the imaging device.
With advances in sensor technology, the use of multispectral imaging for indoor scene acquisition under controlled conditions have also increased. The advantage of multispectral imaging is the ability to acquire more spectral information from a scene, which can be used for spectral reflectance reconstruction [8, 9] of the object's surfaces. Most of the natural objects and surfaces have smooth spectral properties which are helpful for multispectral imaging [10]. With an effective spectral reconstruction from the multispectral data, the device can be used as imaging spectrophotometer and can enable the measurement of device-independent and illuminant-independent image data [11]. Use of multispectral imaging techniques have been proposed for the characterization of imaging devices $[5,12,13]$. The use of digital three channel color cameras as colorimetric devices has been investigated extensively in last decades [14], while multispectral cameras are mostly used for extraction of spectral information. We are interested to also use the multispectral camera as a colorimetric device and obtain the colorimetric information through camera characterization.

Camera characterization involves finding a transform between the camera input space and CIE XYZ color space. Ideally, this transform should be linear and invertible. Such a transform can be found by imaging the surfaces with known spectral reflectance and colorimetric values.

One method for device characterization is to recover the spectral properties of the surfaces being imaged and then compute the tristimulus values from the reconstructed spectral reflectances [5]. There are many methods proposed in the literature for spectral reconstruction. Low dimensional linear models are used in spectral reconstruction from multispectral data with the assumption of smoothness of spectra [8]. The efficiency of a spectral reconstruction system is dependent on the training data and performs well if the test data consist of similar spectral properties. In this work, we investigate the role of training dataset for spectral reconstruction by using non-linear methods. The rest of paper is organized as follows; In Section 2, we provide a brief review of the multispectral imaging system and spectral reconstruction. In Section 3, we explain the experimental framework, discussion of results in Section 4, and followed by conclusions and future aspects. 


\section{Multispectral imaging system}

In a simplified noiseless imaging model, formation of an image depends on the spectral sensitivity of the imaging sensor $m(\lambda)$, the spectral reflectance of the surface $r(\lambda)$ and the spectral power distribution of the illuminant $e(\lambda)$. This formation for the visible wavelength spectrum $\omega$ is defined as

$$
f=\int_{\omega} r(\lambda) e(\lambda) m(\lambda) d \lambda
$$

In practice, we can formulate an extended and discrete version of Eq. 1 as $\mathbf{F}=\mathbf{R E M}$. Considering the spectral sampling of $10 \mathrm{~nm}$ within the wavelength spectrum of $400 \mathrm{~nm}$ to $720 \mathrm{~nm}$ and $K$ number of spectral channels, $\mathbf{F}$ is $S \times N$ matrix ( $S$ is the number of spectral samples being acquired across $N$ wavelengths), $\mathbf{R}$ is $S \times N$ matrix of surface reflectance, $\mathbf{E}$ is the diagonal matrix $(N \times N)$ of the scene illuminant and $\mathbf{M}$ is $N \times K$ matrix, consisting of the spectral sensitivities of the channels.

Spectral reconstruction aims at estimating $\mathbf{R}$ from $\mathbf{F}$. If the term $\mathbf{E M}$ is known and invertible, then the solution is a simple inverse as $\hat{\mathbf{R}}=\mathbf{W F}$, where $\mathbf{W}=\mathbf{E}^{-1} \mathbf{M}^{-1}$ and $\hat{\mathbf{R}}$ is the estimated spectra. Such a transformation does not produce efficient results for spectral reconstruction and the calibration matrix $\mathbf{W}$ is created through a set of measured training spectra. There are many methods for creating $\mathbf{W}$ by using linear methods. One of the methods is through the pseudo inverse, proposed by Maloney and Wandell [10], as;

$$
\mathbf{W}=\mathbf{R}_{t} \mathbf{F}_{t}^{T}\left(\mathbf{F}_{t} \mathbf{F}_{t}^{T}\right)^{-1}
$$

Here the subscript $t$ represents training data, while the superscript $T$ represents the transpose of a matrix.

In most of the spectral reconstruction work, a linear mapping is considered sufficient. There are many methods for determining a linear transform. The idea for using such a transformation is that a few basis functions are helpful in representing the spectral reflectance [10][15]. To determine the basis functions, Principal Component Analysis (PCA) and Karhunen-Loeve transformation is mostly used over a set of measured spectral samples. Imai and Berns [16] proposed the use of principal component analysis over the training data, before using it in Eq. 2. In the pseudo inverse methods proposed in $[10,16]$, the dimensionality of the reflectance model is equal to or less than the number of camera channels [17]. Shi and Healey [13] proposed the use of the first three basis functions separately from the remaining basis functions and trained the calibration matrix W. Wiener estimation [18] is also an efficient linear method for spectral reconstruction. It is defined as;

$$
\mathbf{W}=\mathbf{R}_{t} \mathbf{R}_{t}^{T}(\mathbf{M E})^{T}\left((\mathbf{M E}) \mathbf{R}_{t} \mathbf{R}_{t}^{T}(\mathbf{M E})^{T}+\mathbf{G}\right)^{-1}
$$

Here, $\mathbf{R}_{t} \mathbf{R}_{t}^{T}$ and $\mathbf{G}$ are the autocorrelation matrices of training spectra and additive noise, respectively. $\mathbf{G}$ is in the form of a diagonal matrix consisting of the variance of noise $\sigma^{2}$.
Linear mapping method is computationally efficient and has been used successfully for color camera characterization. As in the analysis provided at [19], three sets of training data were used separately in the training phase and the characterization error is measured after transformation of input data. The three spectral datasets being used for training were ISO-17321-2 [20], Image Engineering dataset and GretagMacbeth ColorChecker. The ISO 17321 data was used to test the characterization. As expected, the error is smallest when the representative data for the test scene was used in the training phase. This suggests that domain specific training data should be used for specific applications. We use the same concept for characterization of a multispectral camera and train the system with different reflectance datasets for comparison of performance and effect of training dataset. If $\mathbf{X}$ is the CIE $\mathrm{XYZ}$ representation of a known spectra $\mathbf{R}$, then the image $\mathbf{F}$ can be transformed into its corresponding colorimetric values through at transformation matrix $\mathbf{W}_{x}$ as;

$$
\mathbf{X}=\mathbf{W}_{x} \mathbf{F}
$$

By using known reflectance spectra and their corresponding colorimetric values, Eq. 4 can be used to find the transformation matrix $\mathbf{W}_{x}$ through the pseudo inverse of $\mathbf{F}$ as;

$$
\mathbf{W}_{x}=\mathbf{X F}^{+}
$$

In this work, we also use non-linear mapping for modelling the mapping between the reflectance spectra and the camera's response. Non-linear methods have been used for spectral reconstruction in previous literature. Ribés and Schmitt [21] used mixture density networks and genetic algorithms for the estimation of spectral reflectance reconstruction of fine art paintings. Artificial neural network is used by Mansouri et al. [22], where the authors formulated the problem of spectral reflectance reconstruction using hetro-associative memories. Yang and Stark [23] proposed the projection onto convex sets, which is an iterative algorithm that projects onto a sequence of constraint sets and eventually produces a solution that satisfies the a-priori constraints. Gómez et al. [24] used a feed-forward back propagation neural network for estimation of spectra in oil paintings. Chane et al. [25] used a neural network, which is trained by using the 15-band multispectral luminescence acquisitions and the corresponding spot spectroscopy luminescence data. Kandi [26] used a neural network to estimate the spectral information of printed samples from camera response data under two illuminants and to investigate the colorimetric characterization of camera. Recently, Hajipour and Nateri [27] used tristimulous values as input to a neural network for recovery of spectral reconstruction. Radial basis functions (RBF) are also used for spectral reconstruction [28, 29]. RBF is used to model the spectral response of camera for the known spectra. In the training phase, $M$ number of clusters are formed by $\mathrm{RBF}$ which aims at minimizing the distance between the similar spectra through the linear least square method [30] and weights $\omega_{i}$ is assigned to each center $c_{i}$. The distance 
being used is Euclidean distance and denoted by $\|$.$\| . A$ dataset of measured spectra and the same samples being captured by the given camera are used to train the model $M$ as;

$$
M=\omega_{o}+\sum_{i=1}^{M} \omega_{i} \phi\left(\left\|\mathbf{f}-c_{i}\right\|\right)
$$

Once the model is trained, it is used to estimate the spectral reflectance of a surface $\hat{\mathbf{r}}$ from the camera input $\mathbf{f}$ as;

$$
\hat{\mathbf{r}}=M(\mathbf{f})
$$

In ISO 17321-2, a procedure for characterization of digital cameras is defined which is achieved through spectral measurements. Sensor spectral sensitivity of the camera and a training set, comprising of measured spectral radiances are used to compute a linear transform. This transform is used to map the input data at the testing stage. In our work, we use a linear method, and also RBF to create a reflection model for a given camera and the training spectra. The reflectance model is used to estimate the reflectance of input camera data and this reflectance is utilized in the measurement of colorimetric characteristics of the camera. Details of the procedure are explained in the following Section.

\section{Experiments}

In the experiments, we use the following spectral datasets;

- ISO 17321 [20] - spectral radiance measurements of 14 natural objects.

- SFU reflectance data [31] - Spectral reflectance of 1995 surfaces.

- Skin colour reflectance database [32] - Spectral measurement of 4392 skin samples taken from 960 people.

- Munsell color chips [33] - Spectral measurement of 1269 surfaces.

- GretagMacbeth ColorChecker - Spectral measurement of 24 patches.

The data being used is in the range of $400 \mathrm{~nm}$ to $700 \mathrm{~nm}$, with $10 \mathrm{~nm}$ spectral sampling. For camera data acquisition, we use spectral sensitivities of two multispectral cameras. One camera is the spectral filter array (SFA) camera [34] and the other is filter wheel multispectral camera from pixelteq [35]. Originally both SFA and pixelteq multispectral cameras are of 8 channels, but since the reflectance data that we have is limited in the spectral range of $400 \mathrm{~nm}$ to $700 \mathrm{~nm}$, we omit the filters in ultra-violet and infra-red wavelength regions. The sensor sensitivities of the simulated filters are shown in Fig. 1. Illuminant D65 is used for creation of radiance data for both training and testing.

In the training phase, we use the simulated multispectral cameras to acquire data from a particular reflectance dataset, which is to be used as training data for camera characterization. As mentioned in Section 2, we use two methods for camera characterization; one is through a linear transform (Eq. 5) and other method is through nonlinear method (RBF). RBF is used to create the reflectance

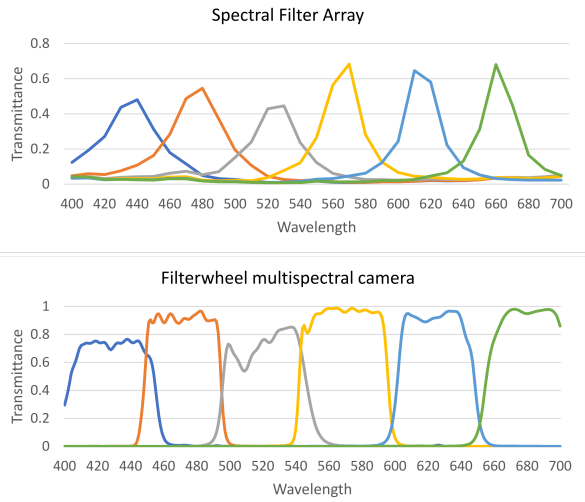

Figure 1: Spectral sensitivities of SFA and filter wheel multispectral cameras used in simulations.

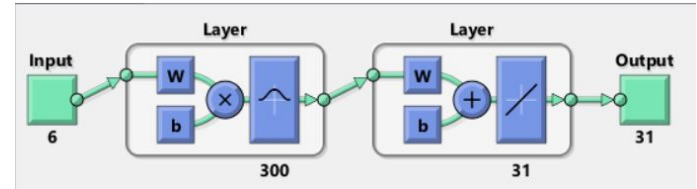

Figure 2: The structure of RBF based network. Input consists of a vector of sensor response (6 in this Fig.) and output is the reconstructed spectrum, consisting of 31 elements. The weights of the hidden layer at input side are adjusted in the training phase till the minimum mean square error is achieved, or maximum number of epochs (300 in our experiments) is reached. The hidden layer at output side consists of 31 nodes and provide the estimation of spectrum for the input camera data.

model for a given set of training spectra and the camera response over that spectra. In the training phase, the weights of hidden layers are adjusted according to the output, until the minimum mean square error is reached. The maximum iterations (epochs) are set to 300 in our experiments. Theoretically, the RBF network create clusters for the input data and the training data by adjusting the weights of the hidden layers. When the test data is given as the input to already trained reflectance model, the output nodes are assigned to the closest matching cluster. There are 31 output nodes which collectively form the output as reflectance spectra from $400 \mathrm{~nm}$ to $700 \mathrm{~nm}$, with $10 \mathrm{~nm}$ sampling. The structure of RBF is shown in Fig. 2.

\section{Evaluation}

To measure the performance of camera characterization, estimated colorimetric values from camera data are compared with the ground truth data. In the linear transform, the output is CIE XYZ values while for the output from $\mathrm{RBF}$, the reconstructed spectra is transformed into XYZ by using the CIE $19312^{\circ}$ Standard Observer and then the XYZ values are converted into CIELAB color space values. For measurement of color difference, we use the CIEDE2000 [36] error metric (also denoted by $\Delta E_{00}$ ). We use the white point of illuminant D65 in the calculation of $\mathrm{XYZ}$ and CIELAB values from the spectra. For each result of $\Delta E_{00}$, the mean, median, maximum and $95^{\text {th }}$ percentile of error is provided. Results are discussed in the following Section. 


\section{Results and Discussion}

Tables 1-10 provide the colorimetric error in terms of $\Delta E_{00}$. Each Table consist of results obtained by using 2 simulated multispectral cameras and a particular reflectance data for training. For each training reflectance dataset, two methods of camera characterization are used; one is the linear method and other is RFB. The odd numbered tables show results from the linear method while even numbered tables contain results obtained from the RBF method.

The ISO 17321 reflectance data consists of 14 spectral reflectances, and the result of using this spectra as a training set is shown in Tables 1 and 2. When the linear method is used, the mean colorimetric error is below one for the skin reflectance dataset and the ISO 17321 reflectance itself, but the performance with SFU reflectance is significantly reduced. The performance of SFA is better than the filter wheel camera in terms of $\Delta E_{00}$. In the case of RBF, Table 2 shows that when the same reflectance data is used as testdata, the error is almost zero, however, when the test data being used is different, then the error values are large. The high values of $\Delta E_{00}$ shows that $\mathrm{RBF}$ is not a good choice for camera characterization when the ISO 17321 reflectance data is used for training.

In Tables 3 and 4, SFU reflectance data is used for training. By using the linear method, the mean of $\Delta E_{00}$ is below 2 for both simulated multispectral cameras. However, it can be seen that the maximum value of error is significantly higher when the same SFU dataset is used as test data. Nonetheless, system trained with the SFU dataset is able to keep the $\Delta E_{00}$ fairly low for all the reflectance datasets being used for testing. Using RBF, $\Delta E_{00}$ for SFU data as test is reduced compared to the linear method and mean error values for Munsell data as test are also below 2 . The reason for the lower error values for Munsell dataset while using SFU reflectances as training is the presence of Munsnell reflectances within the SFU dataset. This can also be seen with the lower error values from ColorChecker reflectances as they are also present in the SFU dataset. However, the results are significantly degraded when ISO 17321 data is used for testing and error values are higher with the RBF technique. The same trend can be seen when skin reflectance data is used for training. Tables 5 and 6 show that with the linear method, there is a lower error of the test spectra while $\Delta E_{00}$ is significantly reduced when the same skin reflectance data is used for training and testing in RBF. However, the error is high in the RBF technique when the test data is not related to training data.

Tables 7-8 consist of $\Delta E_{00}$ obtained when Munsell reflectance data is used for training. These results also follow the same trend and the linear method provides smaller errors compared to RBF. The result from RBF is the best only when the same data is used for training and testing. Tables 9 and 10 consist of results obtained by using ColorChecker reflectance data for training. Once again we see that RBF does not perform better than the linear method, unless the same data is used for training and testing. This trend indicates that when the training data is small, the $\Delta E_{00}$ is near to zero when adequate training data is used.
However, this is a case of over-fitting and does not apply to generic real world scenarios. Also, such small datasets perform the worst when tested with different spectra. This indicates that the training data should have more samples. However, the training samples should have enough relevance to the test spectra and simply increasing the number of training samples do not provide an advantage.

The best result in terms of $\Delta E_{00}$ was obtained when the SFU reflectance dataset was used for training. However, it should be noted that the SFU dataset already consists of the 24 Macbeth color checker patches and 1269 Munsell chips. Additionally, it include reflectance spectra of 120 Dupont paint chips , 170 natural objects , the 350 surfaces in Krinov data set and 57 additional measured surface reflectances. Such a generalized reflectance data is expected to perform well, compared to other datasets. The model trained with SFU dataset is also able to get good results for the ISO 17321 and skin reflectances. This low error indicates that the linear method along with SFU dataset being used for training provides efficient performance, when tested with a simulated multispectral camera.

The results being obtained by using different training spectra provide interesting information about the behavior of a reflectance estimation system. When the training data is highly correlated with the surfaces being tested, the colorimetric characterization can be performed efficiently. This suggests that we can create reflectance data with as many samples as are available. This can avoid the problem of overfitting and underfitting. It is yet to be investigated how much accuracy is required for computer vision applications, which rely on the combination of spectral and colorimetric information.

In order to investigate the camera characterization results on natural scenes, we use SFA for simulation of multispectral image capture on the Foster hyperspectral dataset of natural scenes [37]. These hyperspectral images consist of eight natural scenes from 400 to $720 \mathrm{~nm}$, but we use the data from 400 to $700 \mathrm{~nm}$ since reflectance data for training is available in this region. The reason for using the SFA multispectral camera in simulations is that it is able to provide better results for $\Delta E_{00}$ compared to the filter wheel camera, except in a few cases. Also, we note that since the SFU reflectance data provide better results compared to the other five datasets used for training, we use the SFU data for training the linear model. This model is used to obtain colorimetric information from the multispectral images taken with simulated SFA camera. D65 illumination is used in the simulations. Table 11 show $\Delta E_{00}$ for each image in the Foster dataset. Although mean $\Delta E_{00}$ for these images is relatively low, except for image 1 , the maximum and 95 percentile error values are high. The reason for this error is the absence of relevant spectra in the training dataset. Therefore, if the training spectra consists of a representative spectra and as close to the test images as possible, the error in $\Delta E_{00}$ can be reduced significantly. Nonetheless, the results from Table 11 shows that using linear methods, a multispectral camera can be efficiently characterized for obtaining colorimetric information. 
Table 1: $\Delta E_{00}$ results from simulated SFA and filter wheel cameras on 5 different reflectance datasets. Camera characterization is done through linear transformation of input values and reflectance data from ISO 17321 is used for training.

\begin{tabular}{|c|c|c|c|c|c|c|c|c|c|c|}
\hline \multirow{3}{*}{$\Delta E_{00}$} & \multicolumn{5}{|c|}{ Spectral Filter Array } & \multicolumn{5}{|c|}{ Filterwheel multispectral camera } \\
\hline & \multicolumn{10}{|c|}{ Test data } \\
\hline & ISO 17321 & SFU & Skin Refl. & Munsell & ColorChecker & ISO 17321 & SFU & Skin Refl. & Munsell & ColorChecker \\
\hline Mean & 0.7194 & 3.5423 & 0.946 & 2.6786 & 3.3893 & 0.9883 & 4.196 & 2.0603 & 3.7516 & 5.0281 \\
\hline Median & 0.3213 & 2.7606 & 0.822 & 2.3474 & 2.6463 & 0.4337 & 3.7645 & 1.9961 & 3.7541 & 3.5608 \\
\hline Max & 2.4943 & 20.0891 & 3.2796 & 15.0928 & 8.5842 & 6.1738 & 30.8002 & 5.922 & 27.993 & 15.1954 \\
\hline $95 \%$ & 2.4502 & 10.9307 & 1.8843 & 6.132 & 8.4703 & 5.3738 & 10.0045 & 3.5481 & 7.7155 & 13.3755 \\
\hline
\end{tabular}

Table 2: $\Delta E_{00}$ results from simulated SFA and filter wheel cameras on 5 different reflectance datasets. Colorimetric values from camera input are obtained after spectral reconstruction through RBF, and reflectance data from ISO 17321 is used for training.

\begin{tabular}{|c|c|c|c|c|c|c|c|c|c|c|}
\hline \multirow{3}{*}{$\Delta E_{00}$} & \multicolumn{5}{|c|}{ Spectral Filter Array } & \multicolumn{5}{|c|}{ Filterwheel multispectral camera } \\
\hline & \multicolumn{10}{|c|}{ Test data } \\
\hline & ISO 17321 & SFU & Skin Refl. & Munsell & ColorChecker & ISO 17321 & $\mathrm{SFU}$ & Skin Refl. & Munsell & ColorChecker \\
\hline Mean & 0.0001 & 25.2965 & 16.4351 & 29.7926 & 23.0418 & 0.0001 & 36.6186 & 63.2236 & 40.3671 & 37.0267 \\
\hline Median & 0.0001 & 19.7864 & 10.8621 & 23.6507 & 18.108 & 0.0001 & 32.8391 & 70.8413 & 37.4879 & 33.6008 \\
\hline Max & 0.0001 & 92.9274 & 58.0097 & 99.7072 & 50.2504 & 0.0001 & 141.0209 & 105.3386 & 149.0721 & 93.1658 \\
\hline $95 \%$ & 0.0001 & 59.4169 & 38.551 & 72.0706 & 44.9412 & 0.0001 & 79.3924 & 86.5554 & 84.9848 & 71.3004 \\
\hline
\end{tabular}

Table 3: $\Delta E_{00}$ results from simulated SFA and filter wheel cameras on 5 different reflectance datasets. Camera characterization is done through linear transformation of input values and SFU reflectance data is used for training.

\begin{tabular}{|c|c|c|c|c|c|c|c|c|c|c|}
\hline \multirow{3}{*}{$\Delta E_{00}$} & \multicolumn{5}{|c|}{ Spectral Filter Array } & \multicolumn{5}{|c|}{ Filterwheel multispectral camera } \\
\hline & \multicolumn{10}{|c|}{ Test data } \\
\hline & ISO 17321 & SFU & Skin Refl. & Munsell & ColorChecker & ISO 17321 & SFU & Skin Refl. & Munsell & ColorChecker \\
\hline Mean & 1.7584 & 1.1919 & 0.9249 & 0.8282 & 0.9957 & 1.7431 & 1.2836 & 0.4329 & 0.9254 & 0.958 \\
\hline Median & 1.6313 & 0.832 & 0.9177 & 0.7382 & 0.9537 & 1.8697 & 0.9392 & 0.3338 & 0.8432 & 0.8008 \\
\hline Max & 3.8625 & 11.3672 & 2.0604 & 4.028 & 2.6986 & 2.9034 & 14.8269 & 2.7687 & 7.079 & 2.7164 \\
\hline $95 \%$ & 3.6899 & 3.8758 & 1.3144 & 1.69 & 2.4889 & 2.8989 & 3.6974 & 1.0873 & 1.8486 & 2.2451 \\
\hline
\end{tabular}

Table 4: $\Delta E_{00}$ results from simulated SFA and filter wheel cameras on 5 different reflectance datasets. Colorimetric values from camera input are obtained after spectral reconstruction through RBF, and SFU reflectance data is used for training.

\begin{tabular}{|c|c|c|c|c|c|c|c|c|c|c|}
\hline \multirow{3}{*}{$\Delta E_{00}$} & \multicolumn{5}{|c|}{ Spectral Filter Array } & \multicolumn{5}{|c|}{ Filterwheel multispectral camera } \\
\hline & \multicolumn{10}{|c|}{ Test data } \\
\hline & ISO 17321 & SFU & Skin Refl. & Munsell & ColorChecker & ISO 17321 & SFU & Skin Refl. & Munsell & ColorChecker \\
\hline Mean & 20.3457 & 0.4943 & 5.8507 & 1.4383 & 2.224 & 16.2154 & 0.585 & 6.904 & 1.8177 & 1.6987 \\
\hline Median & 12.6682 & 0.2839 & 4.7344 & 1.3454 & 1.2189 & 6.8432 & 0.3148 & 5.0181 & 1.755 & 1.4933 \\
\hline $\operatorname{Max}$ & 72.5969 & 10.3802 & 38.6426 & 7.9147 & 17.0861 & 111.5009 & 13.781 & 44.2994 & 4.5246 & 4.8049 \\
\hline $95 \%$ & 69.8636 & 1.5687 & 11.3124 & 1.9207 & 9.4372 & 95.7351 & 2.1196 & 17.7355 & 2.2121 & 3.8698 \\
\hline
\end{tabular}

Table 5: $\Delta E_{00}$ results from simulated SFA and filter wheel cameras on 5 different reflectance datasets. Camera characterization is done through linear transformation of input values and skin reflectance dataset is used for training.

\begin{tabular}{|c|c|c|c|c|c|c|c|c|c|c|}
\hline \multirow{3}{*}{$\Delta E_{00}$} & \multicolumn{5}{|c|}{ Spectral Filter Array } & \multicolumn{5}{|c|}{ Filterwheel multispectral camera } \\
\hline & \multicolumn{10}{|c|}{ Test data } \\
\hline & ISO 17321 & SFU & Skin Refl. & Munsell & ColorChecker & ISO 17321 & SFU & Skin Refl. & Munsell & ColorChecker \\
\hline Mean & 2.1332 & 1.8638 & 0.1564 & 1.1973 & 1.4295 & 2.189 & 1.9801 & 0.2592 & 1.3363 & 1.7026 \\
\hline Median & 1.9484 & 1.2062 & 0.1298 & 1.0235 & 1.103 & 2.3253 & 1.3586 & 0.2128 & 1.1818 & 1.4986 \\
\hline Max & 7.2685 & 13.5945 & 1.3601 & 5.1716 & 4.8582 & 5.2003 & 21.4672 & 2.26 & 8.4396 & 3.9487 \\
\hline $95 \%$ & 6.4475 & 7.1785 & 0.3684 & 2.5389 & 4.1924 & 5.0175 & 6.2132 & 0.6132 & 2.8947 & 3.6536 \\
\hline
\end{tabular}

Table 6: $\Delta E_{00}$ results from simulated SFA and filter wheel cameras on 5 different reflectance datasets. Colorimetric values from camera input are obtained after spectral reconstruction through RBF, and skin reflectance dataset is used for training.

\begin{tabular}{|c|c|c|c|c|c|c|c|c|c|c|}
\hline \multirow{3}{*}{$\Delta E_{00}$} & \multicolumn{5}{|c|}{ Spectral Filter Array } & \multicolumn{5}{|c|}{ Filterwheel multispectral camera } \\
\hline & \multicolumn{10}{|c|}{ Test data } \\
\hline & ISO 17321 & SFU & Skin Refl. & Munsell & ColorChecker & ISO 17321 & SFU & Skin Refl. & Munsell & ColorChecker \\
\hline Mean & 19.1457 & 10.6788 & 0.1257 & 11.4708 & 17.4727 & 14.8275 & 19.4485 & 0.2013 & 19.8317 & 23.2347 \\
\hline Median & 11.6453 & 7.096 & 0.1033 & 7.7014 & 14.4252 & 5.7423 & 13.0797 & 0.1617 & 13.2395 & 16.1735 \\
\hline Max & 72.6046 & 112.8374 & 0.7225 & 69.1411 & 33.0798 & 77.0516 & 171.6711 & 1.0909 & 168.5628 & 103.2295 \\
\hline $95 \%$ & 66.613 & 29.3613 & 0.3024 & 31.4903 & 32.5279 & 67.9785 & 54.0677 & 0.4997 & 55.8913 & 89.5829 \\
\hline
\end{tabular}

Table 7: $\Delta E_{00}$ results from simulated SFA and filter wheel cameras on 5 different reflectance datasets. Camera characterization is done through linear transformation of input values and munsell reflectance dataset is used for training.

\begin{tabular}{|c|c|c|c|c|c|c|c|c|c|c|}
\hline \multirow{3}{*}{$\Delta E_{00}$} & \multicolumn{5}{|c|}{ Spectral Filter Array } & \multicolumn{5}{|c|}{ Filterwheel multispectral camera } \\
\hline & \multicolumn{10}{|c|}{ Test data } \\
\hline & ISO 17321 & SFU & Skin Refl. & Munsell & ColorChecker & ISO 17321 & SFU & Skin Refl. & Munsell & ColorChecker \\
\hline Mean & 2.145 & 1.3519 & 2.0956 & 0.7224 & 0.9969 & 1.8357 & 1.5966 & 0.6957 & 0.8778 & 1.0108 \\
\hline Median & 2.0369 & 0.7722 & 2.0932 & 0.6355 & 0.6841 & 1.7431 & 0.8409 & 0.6622 & 0.7276 & 0.6461 \\
\hline $\operatorname{Max}$ & 4.1234 & 12.0243 & 3.9997 & 3.7152 & 2.7537 & 4.1763 & 25.4742 & 2.9312 & 11.4406 & 3.1553 \\
\hline $95 \%$ & 3.9508 & 5.2581 & 2.7588 & 1.5458 & 2.5626 & 3.8847 & 6.2594 & 1.0421 & 1.9906 & 2.5951 \\
\hline
\end{tabular}


Table 8: $\Delta E_{00}$ results from simulated SFA and filter wheel cameras on 5 different reflectance datasets. Colorimetric values from camera input are obtained after spectral reconstruction through RBF, and munsell reflectance dataset is used for training.

\begin{tabular}{|c|c|c|c|c|c|c|c|c|c|c|}
\hline \multirow{3}{*}{$\Delta E_{00}$} & \multicolumn{5}{|c|}{ Spectral Filter Array } & \multicolumn{5}{|c|}{ Filterwheel multispectral camera } \\
\hline & \multicolumn{10}{|c|}{ Test data } \\
\hline & ISO 17321 & SFU & Skin Refl. & Munsell & ColorChecker & ISO 17321 & SFU & Skin Refl. & Munsell & ColorChecker \\
\hline Mean & 24.0039 & 2.9209 & 9.3015 & 0.1884 & 2.1101 & 15.5738 & 5.1576 & 11.7369 & 0.2483 & 1.9564 \\
\hline Median & 14.5737 & 1.3599 & 8.448 & 0.1334 & 1.2064 & 8.6756 & 1.7684 & 10.8623 & 0.1651 & 1.2751 \\
\hline $\operatorname{Max}$ & 110.9224 & 76.2363 & 34.1916 & 2.9005 & 8.2254 & 80.9067 & 99.7878 & 41.0444 & 1.9762 & 8.6787 \\
\hline $95 \%$ & 98.0849 & 8.8584 & 15.3718 & 0.5308 & 7.9414 & 70.9825 & 24.3242 & 19.2322 & 0.7876 & 6.7308 \\
\hline
\end{tabular}

Table 9: $\Delta E_{00}$ results from simulated SFA and filter wheel cameras on 5 different reflectance datasets. Camera characterization is done through linear transformation of input values, and reflectance data from 24 patches of ColorChecker is used for training.

\begin{tabular}{|c|c|c|c|c|c|c|c|c|c|c|}
\hline \multirow{3}{*}{$\Delta E_{00}$} & \multicolumn{5}{|c|}{ Spectral Filter Array } & \multicolumn{5}{|c|}{ Filterwheel multispectral camera } \\
\hline & \multicolumn{10}{|c|}{ Test data } \\
\hline & ISO 17321 & SFU & Skin Refl. & Munsell & ColorChecker & ISO 17321 & SFU & Skin Refl. & Munsell & ColorChecker \\
\hline Mean & 1.7404 & 1.6828 & 1.0393 & 1.2164 & 1.0956 & 2.204 & 2.197 & 0.6699 & 1.7178 & 1.1093 \\
\hline Median & 1.7835 & 1.142 & 1.0493 & 1.0405 & 0.7157 & 2.2295 & 1.5898 & 0.5744 & 1.4805 & 0.9898 \\
\hline Max & 3.3151 & 12.749 & 2.4795 & 4.2034 & 3.2838 & 3.8507 & 19.9727 & 3.8322 & 11.5692 & 3.0324 \\
\hline $95 \%$ & 3.2561 & 5.7123 & 1.5598 & 2.6905 & 2.4682 & 3.7146 & 6.5214 & 1.5944 & 3.74 & 2.6195 \\
\hline
\end{tabular}

One of the limitations of the proposed spectral reconstruction framework is that the camera data should be taken in the same illumination under which the system is trained. This condition can be fulfilled when the data acquisition is performed in controlled conditions and the illumination is measured during the experiments. However, measurement of illumination by a telespectroradiometer in outdoor scenes is not always feasible. In the case of a change in illumination, the system should be trained again with the new illumination, and it must be made sure that the same illumination was present during the test image acquisition as well. To overcome this limitation, the concept of multispectral constancy was recently proposed [38]. In this method, the estimation of illumination in multispectral images [39] is performed after the acquisition, and the effect of illumination is removed from the multispectral data. The spectral reconstruction system is trained/calibrated with the training data acquired with the same camera under a canonical illuminant. The acquired image is also transformed into a canonical representation and then is provided as input to the spectral reconstruction system. With an efficient multispectral constancy and spectral reconstruction system, use of the multispectral camera can be increased widely for imaging in uncontrolled environments.

\section{Conclusion}

In this paper, we analyse the role of training data for obtaining colorimetric information from two simulated multispectral cameras. We use a linear method for direct conversion from multispectral data into colorimetric data, and a spectral reconstruction method whereby the spectral estimation is performed first and this information is subsequently used to obtain colorimetric data. For spectral reconstruction, a Radial basis function based network is trained to create a model of reflectance and the input data from the multispectral camera is used to obtain the spectral reconstruction. The training data plays an important role in defining the efficiency of the camera characterization. We investigated the reflectance models created with reflectance spectra from ISO 17321, GretagMacbeth
ColorChecker, Skin reflectance, Munsell chips and SFU dataset. The SFU dataset consists of reflectance spectra from a number of objects including paints, natural objects, color patches and dyes. The generic nature of this dataset makes it a good training data for the spectral reflectance reconstruction system. We found that by using a linear method, efficient colorimetric information can be obtained and the results outperform the non-linear method being tested. The results obtained are promising and now further work is required to validate these findings by using the real camera and imaging environment.

\section{References}

[1] B. A. Wandell and J. E. Farrell, "Water into wine: converting scanner RGB to tristimulus xyz," Proc. SPIE, , Device-Independent Color Imaging and Imaging Systems Integration, vol. 1909, pp. 92-101, 1993.

[2] J. Farrell, D. Sherman, and B. W, "How to turn your scanner into a colorimeter," in Proc. of ISET T 10th Int. Congress on Adv. in Non-Impact Printing Technologies, pp. 579-581, 1994.

[3] T. Johnson, "Methods for characterizing colour scanners and digital cameras," Displays, vol. 16, no. 4, pp. $183-191,1996$.

[4] W. Wu, J. P. Allebach, and M. Analoui, "Imaging colorimetry using a digital camera," Journal of Imaging Science and Technology, vol. 44, pp. 267-279, 2000.

[5] G. D. Finlayson and P. M. Morovic, "Metamer constrained color correction," Journal of Imaging Science and Technology, vol. 44, no. 4, pp. 295-300, 2000.

[6] G. Hong, M. R. Luo, and P. A. Rhodes, "A study of digital camera colorimetric characterization based on polynomial modeling," Color Research 8 Application, vol. 26 , no. 1 , pp. 76-84, 2001.

[7] F. H. Imai, S. Quan, M. R. Rosen, and R. S. Berns, "Digital camera filter design for colorimetric and spectral accuracy," in Proc. of third international confer- 
Table 10: $\Delta E_{00}$ results from simulated SFA and filter wheel cameras on 5 different reflectance datasets. Colorimetric values from camera input are obtained after spectral reconstruction through RBF, and reflectance data from 24 patches of ColorChecker is used for training.

\begin{tabular}{|c|c|c|c|c|c|c|c|c|c|c|}
\hline \multirow{3}{*}{$\Delta E_{00}$} & \multicolumn{5}{|c|}{ Spectral Filter Array } & \multicolumn{5}{|c|}{ Filterwheel multispectral camera } \\
\hline & \multicolumn{10}{|c|}{ Test data } \\
\hline & ISO 17321 & SFU & Skin Refl. & Munsell & ColorChecker & ISO 17321 & SFU & Skin Refl. & Munsell & ColorChecker \\
\hline Mean & 23.0606 & 6.7614 & 10.5938 & 5.0789 & 0.0001 & 16.5937 & 6.7808 & 8.7854 & 3.7128 & 0.0001 \\
\hline Median & 14.3294 & 4.6457 & 8.821 & 4.0598 & 0.0001 & 8.0794 & 3.0843 & 7.7112 & 2.5198 & 0.0001 \\
\hline Max & 79.9477 & 65.128 & 37.6771 & 33.2546 & 0.0001 & 67.2223 & 122.6417 & 33.1485 & 31.1151 & 0.0001 \\
\hline $95 \%$ & 75.5436 & 24.2184 & 21.3308 & 12.9415 & 0.0001 & 62.5552 & 23.1108 & 15.4279 & 10.8399 & 0.0001 \\
\hline
\end{tabular}

Table 11: $\Delta E_{00}$ results from simulated SFA on the Foster dataset of hyperspectral reflectance images. Camera characterization is done through linear transformation of input values and SFU reflectance data is used for training.

\begin{tabular}{cccllllll}
\hline \multirow{2}{*}{$\Delta E_{00}$} & \multicolumn{7}{c}{ Reflectance images from Foster hyperspectral data } \\
\cline { 2 - 8 } & Img 1 & Img 2 & Img 3 & Img 4 & Img 5 & $\operatorname{Img} 6$ & $\operatorname{Img} 7$ & $\operatorname{Img~8}$ \\
\hline Mean & 7.3972 & 1.7079 & 3.7541 & 2.1279 & 1.3803 & 3.2125 & 3.5741 & 4.8736 \\
\hline Median & 6.1865 & 1.2901 & 1.9380 & 1.2586 & 1.1031 & 3.0211 & 3.2730 & 2.8670 \\
\hline Max & 34.2251 & 39.2696 & 82.2016 & 39.8810 & 20.9365 & 28.1000 & 16.6525 & 35.2226 \\
\hline $\mathbf{9 5 \%}$ & 16.1055 & 4.0502 & 13.6360 & 7.1016 & 3.3286 & 6.2330 & 6.9585 & 17.9389 \\
\hline
\end{tabular}

ence on multispectral color science, pp. 13-16, University of Joensuu, Finland, 2001.

[8] J. Y. Hardeberg, Acquisition and Reproduction of Color Images: Colorimetric and Multispectral Approaches. Parkland, FL: Dissertation.com, 2001.

[9] D. Connah, J. Y. Hardeberg, and S. Westland, "Comparison of linear spectral reconstruction methods for multispectral imaging," in International Conference on Image Processing, ICIP, vol. 3, pp. 1497-1500, 2004 .

[10] L. T. Maloney, "Evaluation of linear models of surface spectral reflectance with small numbers of parameters," J. Opt. Soc. Am. A, vol. 3, pp. 1673-1683, Oct 1986.

[11] V. Cheung, C. Li, J. Hardeberg, D. Connah, and S. Westland, "Characterization of trichromatic color cameras by using a new multispectral imaging technique," J. Opt. Soc. Am. A, vol. 22, pp. 1231-1240, Jul 2005.

[12] R. S. Berns and M. J. Shyu, "Colorimetric characterization of a desktop drum scanner using a spectral model," Journal of Electronic Imaging, vol. 4, no. 4, pp. 360-372, 1995.

[13] M. Shi and G. Healey, "Using reflectance models for color scanner calibration," J. Opt. Soc. Am. A, vol. 19, pp. 645-656, Apr 2002.

[14] V. Cheung, S. Westland, D. Connah, and C. Ripamonti, "A comparative study of the characterisation of colour cameras by means of neural networks and polynomial transforms," Coloration Technology, vol. 120, no. 1, pp. 19-25, 2004.

[15] T. Jaaskelainen, J. Parkkinen, and S. Toyooka, "Vector-subspace model for color representation," J. Opt. Soc. Am. A, vol. 7, pp. 725-730, Apr 1990.
[16] F. H. Imai and R. S. Berns, "Spectral estimation using trichromatic digital cameras," in Proceedings of the International Symposium on Multispectral Imaging and Color Reproduction for Digital Archives, vol. 42, pp. 1-8, Chiba University Chiba, Japan, 1999.

[17] D. Connah, S. Westland, and M. G. A. Thomson, "Recovering spectral information using digital camera systems," Coloration Technology, vol. 117, no. 6, pp. 309-312, 2001.

[18] H.-L. Shen, P.-Q. Cai, S.-J. Shao, and J. H. Xin, "Reflectance reconstruction for multispectral imaging by adaptive wiener estimation," Opt. Express, vol. 15, pp. 15545-15554, Nov 2007.

[19] International Color Consortium, "Digital camera spectral calibration," 2017. http://www.color.org/ resources/photography/spectral_calibration. xalter.

[20] ISO/TR 17321-2:2012, "Graphic technology and photography - Colour characterization of digital still cameras (DSCs)." https://www.iso.org/standard/ 58003.html.

[21] A. Ribés and F. Schmitt, "A fully automatic method for the reconstruction of spectral reflectance curves by using mixture density networks," Pattern Recognition Letters, vol. 24, no. 11, pp. 1691 - 1701, 2003. Colour Image Processing and Analysis. First European Conference on Colour in Graphics, Imaging, and Vision.

[22] A. Mansouri, F. S. Marzani, and P. Gouton, "Neural networks in two cascade algorithms for spectral reflectance reconstruction," in IEEE International Conference on Image Processing, vol. 2, pp. II-718-21, Sept 2005.

[23] Y. Yang and H. Stark, "Solutions of several colormatching problems using projection theory," J. Opt. Soc. Am. A, vol. 11, pp. 89-96, Jan 1994. 
[24] C. Osorio-Gómez, E. Mejía-Ospino, and J. GuerreroBermúdez, "Spectral reflectance curves for multispectral imaging, combining different techniques and a neural network," Revista mexicana de física, vol. 55, no. 2, pp. 120-124, 2009.

[25] C. S. Chane, M. Thoury, A. TourniÃl, and J.-P. Echard, "Implementation of a neural network for multispectral luminescence imaging of lake pigment paints," Applied Spectroscopy, vol. 69, no. 4, pp. 430$441,2015$.

[26] S. G. Kandi, "Estimating spectral and colorimetric data of printed samples from digital camera responses under two illuminants by neural network," Journal of Printing Science and Technology, vol. 47, no. 6, pp. 392-400, 2010.

[27] A. Hajipour and A. Shams-Nateri, "Effect of classification by competitive neural network on reconstruction of reflectance spectra using principal component analysis," Color Research $\&$ Application, vol. 42, no. 2, pp. 182-188, 2017.

[28] M. D. Buhmann, Radial basis functions: theory and implementations, vol. 12. Cambridge university press, 2003.

[29] R. M. H. Nguyen, D. K. Prasad, and M. S. Brown, Training-Based Spectral Reconstruction from a Single $R G B$ Image, pp. 186-201. Cham: Springer International Publishing, 2014.

[30] S. Chen, C. F. N. Cowan, and P. M. Grant, "Orthogonal least squares learning algorithm for radial basis function networks," IEEE Transactions on Neural Networks, vol. 2, pp. 302-309, Mar 1991.

[31] K. Barnard, L. Martin, B. Funt, and A. Coath, "A data set for color research," Color Research $\&$ Application, vol. 27, no. 3, pp. 147-151, 2002.

[32] K. Xiao, J. M. Yates, F. Zardawi, S. Sueeprasan, N. Liao, L. Gill, C. Li, and S. Wuerger, "Characterising the variations in ethnic skin colours: a new calibrated data base for human skin," Skin Research and Technology, vol. 23, no. 1, pp. 21-29, 2017.

[33] University of Joensuu, "Database - Munsell Colors Matt (Spec)." http://cs.joensuu.fi/spectral/ databases/download/munsell_spec_matt.htm.

[34] P.-J. Lapray, X. Wang, J.-B. Thomas, and P. Gouton, "Multispectral filter arrays: Recent advances and practical implementation," Sensors, vol. 14, no. 11, pp. 21626-21659, 2014.

[35] "SpectroCam ${ }^{\mathrm{TM}}$ Multispectral Wheel Cameras." https://pixelteq.com/spectrocam/.

[36] CIE, Improvement to industrial colour-difference evaluation. CIE technical report 142-2001, Vienna: Central Bureau of the CIE, 2001.
[37] D. H. Foster, K. Amano, S. M. C. Nascimento, and M. J. Foster, "Frequency of metamerism in natural scenes," J. Opt. Soc. Am. A, vol. 23, p. 2359, oct 2006.

[38] H. A. Khan, J. B. Thomas, and J. Y. Hardeberg, "Multispectral constancy based on spectral adaptation transform," in 20th Scandinavian Conference on Image Analysis, Tromsø, Norway (P. Sharma and F. M. Bianchi, eds.), pp. 459-470, Springer International Publishing, June 12-14, 2017.

[39] H. A. Khan, J. B. Thomas, J. Y. Hardeberg, and O. Laligant, "Illuminant estimation in multispectral imaging," J. Opt. Soc. Am. A, vol. 34, no. 6, 2017. 